\title{
Comparative Evaluation of Chlorpyrifos Exposure Estimates from Whole-body Dermal Dosimetry and Urinary Trichloro-2- pyridinol (TCP) Methods
}

\author{
Albert Atabila ${ }^{\mathrm{a}}$, Dung Tri Phung ${ }^{\mathrm{a}}$, Ross Sadler ${ }^{\mathrm{a}}$, Des Connell ${ }^{\mathrm{a}}$ and Cordia Chu ${ }^{\mathrm{a}}$ \\ ${ }^{a}$ Centre for Environment and Population Health, Griffith School of Environment, Griffith \\ University, 170 Kessel Road, Nathan, QLD 4111, Australia.
}

\section{Abstract}

Chlorpyrifos is one of the most widely used organophosphate pesticides and has a record of adverse effects on applicators. Assessment of exposure to chlorpyrifos based on its urinary metabolite, 3,5,6-trichloro-2-pyridinol (TCP), is considered as the most accurate. However, urine sampling can be difficult, and the laboratory analytical procedures involved are complex and expensive. A simpler approach for assessing pesticide exposure among applicators is the whole-body dermal dosimetry method, but this needs validation. The objective of this study was to compare chlorpyrifos exposure estimates obtained separately with the urinary TCP and the whole-body dermal dosimetry methods from applicators. Exposure estimates from the whole-body dermal dosimetry method (5 to $29 \mu \mathrm{g} / \mathrm{kg} /$ day) showed less variation than those from the urinary TCP method ( 1 to $71 \mu \mathrm{g} / \mathrm{kg} /$ day), but both were in close agreement at the mean level (16 $\mu \mathrm{g} / \mathrm{kg} /$ day and $15 \mu \mathrm{g} / \mathrm{kg} /$ day, respectively). The whole-body dermal dosimetry method is therefore valid for providing estimates of the typical levels of pesticide exposure among applicators in situations where the urinary TCP method cannot be applied. 
Chlorpyrifos is an important organophosphate pesticide used extensively throughout the world that has been associated with adverse health effects among applicators (Garabrant et al., 2009; Farahat et al., 2011; Khan et al., 2014). Exposure to chlorpyrifos among applicators has usually been assessed based on the metabolite, 3,5,6-trichloro-2-pyridinol (TCP) found in the urine of exposed persons (Saieva et al., 2004; Baker et al., 2005; Phung et al., 2012b; Atabila et al., $2018 \mathrm{~b}$ ). The use of urinary TCP for evaluating chlorpyrifos exposure is widely accepted because the method gives a measure of the actual absorbed dose due to exposure (Albertini et al., 2006; Ellison et al., 2012; Fustinoni et al., 2014). The method also takes into account exposures from all routes and therefore presents an overall measure of exposure (Sexton et al., 2004; Albertini et al., 2006; Barr and Angerer, 2006). However, urine sampling can be difficult for some individuals and the laboratory analysis procedures involved are often complex and expensive (Fenske and Day, 2005; Putnam and Clark, 2007; Scher et al., 2007).

The dermal route is the most important pathway by which pesticide applicators are exposed with the back-pack spraying method of application (Dowling and Seiber, 2002; Damalas and Eleftherohorinos, 2011; Fenske et al., 2012). This route may account for about $94-96 \%$ of method of evaluating chlorpyrifos exposure (An et al., 2014; Atabila et al., 2017) may therefore be employed in many situations as an alternative to the urinary TCP method. The whole-body dermal dosimetry method involves the use of absorbent coveralls, hand gloves, socks and skin wipes to intercept pesticide residues that would potentially reach and/or penetrate the skin of applicators during spraying events. Studies have been carried to assess the accuracy of the 
2004; Fenske and Day, 2005; Ross et al., 2008). In these studies, whole-body dermal dosimetry and urinary TCP methods were carried out concurrently for each worker for the same exposure event, and the resulting absorbed dose estimates were compared. While these studies provide useful information on how the absorbed dose estimates of chlorpyrifos from the whole-body dermal dosimetry and urinary TCP methods compare with one another, the study designs applied result in the dose estimates for each of the two methods being incomplete. For instance, with the dose estimate from the urinary TCP method, the concurrent dermal dosimeter method results in interception of some quantity of insecticides that would have entered the body of the workers and subsequently eliminated as urinary TCP. This situation can make comparison of the estimated absorbed dose from the two methods problematic. Ideally, each of the two methods should be applied separately and for separate exposure events for comparison of the resulting dose estimates.

We have previously assessed the levels of exposure to chlorpyrifos with rice farmers in Ghana, using the whole-body dermal dosimetry and urinary TCP methods (Atabila et al., 2017; Atabila et al., 2018a; Atabila et al., 2018b). With each applicator, the whole-body dermal dosimetry and urinary TCP methods were carried out separately to evaluate similar exposure events. Thus, the level of chlorpyrifos exposure during each event was fully assessed with either the wholebody dermal dosimetry method or the urinary TCP method, in contrast to previous studies (Geer et al., 2004; Fenske and Day, 2005; Ross et al., 2008), as outlined above. The objective of the present study was to carry out a comparative evaluation of chlorpyrifos exposure estimates from the whole-body dermal dosimetry and urinary TCP methods utilizing the results obtained in the previous investigations (Atabila et al., 2017; Atabila et al., 2018a; Atabila et al., 2018b). 


\subsection{Methods}

The details of the study setting, participants, data collection procedures, laboratory analytical procedures, absorbed dose estimation have been previously described (Atabila et al., 2017; Atabila et al., 2018a; Atabila et al., 2018b). Ethical clearance for the study was obtained from both Ghana Health Service Ethical Review Committee (GHS-ERC: 10/07/15) and Griffith University Human Ethics Committee (ENV/24/15/HREC).

\subsection{Study Setting and Participants}

The study was conducted among small scale $(<2$ ha) rice farmers from a rural farming community located at south-eastern part of Ghana. Chlorpyrifos (480g/L Emulsifiable Concentrate - 480 EC) is regularly applied by the farmers, with the use of hand-pressurized knapsack spraying devices carried on the back. The spraying activities in this study were all carried out in the morning between the hours of 6 to 8 am, when the temperature was generally cooler, and the wind was calm.

\subsection{Whole-body Dermal Dosimetry Method}

\subsubsection{Sampling Procedure}

The whole-body dermal dosimetry method carried out in this study was for one chlorpyrifos spray event for each applicator. The method was based on the whole-body dermal dosimetry protocol of the World Health Organisation (1982) and the Organisation for Economic Cooperation and Development (OECD, 1997). During the day of spraying, the applicators were initially dressed in a new set of sampling suit which consisted of Tyvek under-wear garment, white cotton hand gloves and socks. The applicators subsequently wore their usual farm clothes 
over the sampling suit before starting pesticide spraying activities. The farm clothes of the applicators mainly consisted of cotton-made short-sleeve shirts and long pants. Only two of the applicators used Personal Protective Equipment (PPE), which was safety glasses. After the spraying event, the sampling suit of each applicator was immediately removed, packed and transported in a sample container with ice to the Pesticides Residues Laboratory of Ghana Standards Authority for analysis (Atabila et al., 2017; Atabila et al., 2018a).

\subsubsection{Laboratory Analytical Procedure}

The extraction and laboratory analysis of the sampling suit for chlorpyrifos were performed with a modified version of the agricultural chemicals analytical procedures of the Department of Food Safety of Japan (DFS, 2006). A Varian CP 3800 Gas Chromatograph (Varian Associates Inc, USA) equipped with Pulsed Flame Photometric Detector (PFPD) and a CombiPAL auto sampler was used to analyse the extract obtained from the sampling suits (Atabila et al., 2017; Atabila et al., 2018a).

\subsubsection{Chlorpyrifos Absorbed Daily Dose Estimation}

The Absorbed Daily Dose (ADD ${ }_{\mathrm{DEM}}$ ) of chlorpyrifos from dermal exposure for each applicator was estimated using the following equation, adapted from Health Canada (2014): 
where, TDE is Total Dermal Exposure ( $\mu \mathrm{g} /$ day), obtained as the total mass of chlorpyrifos residues extracted from the sampling suit for each applicator for a day's spray event; DAF, Dermal Absorption Factor (4.3 \%) (Meuling et al., 2005); and BW, Body Weight of each applicator $(\mathrm{kg})$. A rational for using a DAF of $4.3 \%$ is provided in Atabila et al. (2018a).

\subsection{Urinary TCP Method}

\subsubsection{Sampling Procedure}

Similar to the whole-body dermal dosimetry method, the urinary TCP method was carried out for one chlorpyrifos spray event for each applicator. Prior to each sampling day, each applicator was provided with a set of sampling items, that consisted of an ice chest $(8 \mathrm{~L})$, ice packs and a plastic jar (2 L). Each applicator provided six 24-hour (or whole day) urine samples over a period of six days (one sample per day). These included one sample before the application day (background sample), one sample during the application day and four samples after the application day (post-application samples). Three aliquots were prepared from each sample and stored in High-density Polyethylene (HDPE) bottles $(60 \mathrm{~mL})$ for laboratory analysis (Atabila et al., 2018b).

\subsubsection{Laboratory Analytical Procedure}

Aliquots of the urine $(1 \mathrm{~mL})$ were spiked with isotopically labelled TCP, treated with $10 \mathrm{M}$ $\mathrm{NaOH}(\mathrm{pH}>12)$ and hydrolysed at $60^{\circ} \mathrm{C}$ for two hours. The samples were then adjusted to $\mathrm{pH}$ $<3$ with $42.5 \% \mathrm{w} / \mathrm{w} \mathrm{H}_{3} \mathrm{PO}_{4}$ and $0.45 \mu \mathrm{m}$ filtered. The prepared samples were subsequently analysed using Liquid Chromatography Tandem Mass Spectrometry (LC-MS/MS) in positive ESI mode on a Shimadzu Prominence UFLC (Kyoto, Japan), coupled to an Applied Biosystems API 4000 mass spectrometer (Framingham, USA) with a Kinetex C18 column (50x2.1mm, 
$1415 \mu \mathrm{m}$, Phenomenex, Torrance, USA) and a $1 \%$ to $95 \%$ methanol gradient with $0.1 \%$ acetic acid.

142 The analytical quality control consisted of duplicate samples, spiked samples, conjugate spiked samples, blank samples, spiked blank samples and conjugate spiked blank samples (Atabila et al., 2018b).

To compensate for urine volume excretion variation with the applicators, urinary creatinine concentrations were determined and applied to normalize the urinary TCP concentrations of the applicators (Barr et al., 2005; Cocker et al., 2011). The creatinine concentrations were determined based on the kinetic Jaffe reaction colorimetric method (Mohabbati-Kalejahi et al., 2012), with the use of a Mindray BS-120 automated analyzer (Shenzhen Mindray Bio-Medical Electronics Co., Ltd). The analytical quality control consisted of duplicate sample analysis (Atabila et al., 2018b).

\subsubsection{Chlorpyrifos Absorbed Daily Dose Estimation}

Using the post-application urinary TCP levels, the levels of Absorbed Daily Dose of chlorpyrifos from application exposure $\left(\mathrm{ADD}_{\mathrm{TCP}}\right)$ were calculated from the equation below (Fenske et al., 2012; Phung et al., 2012b):

where, $\mathrm{C}$ is the concentration of urinary TCP excreted per day ( $\mu \mathrm{g} / \mathrm{g}$ creatinine); $\mathrm{Cn}$, expected mass of creatinine excreted per day(g/day); CF, correction factor of 100/70 for urinary TCP (about $70 \%$ of chlorpyrifos is excreted as TCP in urine ) (Nolan et al., 1984); $\mathrm{MW}_{\mathrm{CPF}}$, the 
molecular weight of chlorpyrifos $(350.6 \mathrm{~g} / \mathrm{mol})$; $\mathrm{MW}_{\mathrm{TCP}}$, the molecular weight of $\mathrm{TCP}$ (198.5g/mol); and BW, the body weight of each applicator $(\mathrm{kg})$.

\subsection{Data Analysis}

In all, 24 applicators participated in the whole-body dermal dosimetry method, whereas 21 applicators participated in the urinary TCP method. However, sixteen of the applicators were involved in both methods. Only the results of this group were analysed in the current study.

\subsubsection{Test of Difference in Field Factors and Chlorpyrifos Exposure Estimates between the Whole-body Dermal Dosimetry and Urinary TCP Methods}

Since the whole-body dermal dosimetry and urinary TCP methods were undertaken during separate exposure events, differences in the field factors between the events could impact on the exposure dose estimates. To investigate this, the non-parametric Wilcoxon signed ranks test was employed to test for differences in medians of non-normally distributed continuous variables (quantity of insecticide applied, farm size and crop height) between the two methods. A normally distributed continuous variable (spraying duration), on the other hand, was investigated using the parametric paired samples t-test on the means. For categorical variables (use of PPE, type of shirt, type of trouser, occurrences of leaky spray tank and insecticide spillage), the non-parametric McNemar test was used to test for differences in proportions. Also, the difference in the means of the exposure estimates between the whole-body dermal dosimetry and urinary TCP methods were evaluated with Wilcoxon signed ranks test. The analysis was performed using the Statistical Package for the Social Sciences (SPSS) program (Version 20). 
2.4.2 Cumulative Probability Distribution Plots of Chlorpyrifos Exposure Estimates from the Whole-body Dermal Dosimetry and Urinary TCP Methods

The distributions of the exposure estimates from the whole-body dermal dosimetry and urinary TCP methods were assessed with Cumulative Probability Distribution (CPD) plots. The data of the exposure estimates were ranked from the lowest to the highest. The Cumulative Probability (CP) of each data point was then calculated using the equation below:

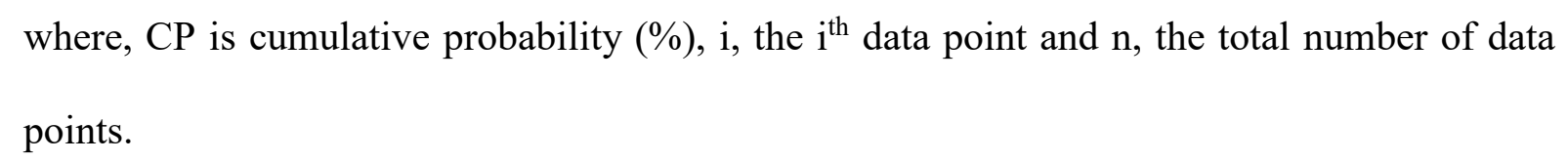
points.

Using the data points and the CP values, the CPD of the exposure estimates were subsequently plotted.

\subsection{Results and Discussion}

\subsection{Comparison of the Field Factors of the Whole-body Dermal Dosimetry and} Urinary TCP Methods

Comparison of the field factors evaluated during both the whole-body dermal dosimetry and urinary TCP methods comprises a validation that the exposure estimates of the two methods can be directly compared. The field factors recorded during the dermal and urinary TCP methods are shown in Table 1. Also shown are the p-values of the statistical tests carried out to investigate differences in the field factors between the two exposure methods. Out of the 
nine field factors investigated, only two factors (farm size and spraying duration) differed significantly between the whole-body dermal dosimetry and urinary TCP methods ( 0.4 ha and 0.6 ha; $53 \mathrm{~min}$ and $79 \mathrm{~min}$, respectively; $\mathrm{p}<0.05$ ). It may therefore be assumed that the field factors of the dermal and biomonitoring methods were similar, especially, when the quantities of insecticide formulation applied during the two methods were not statistically different (156 $\mathrm{mL}$ and $162 \mathrm{~mL}$, respectively; $\mathrm{p}>0.05$ ). Compared to other field factors, quantity of pesticide applied has consistently been identified as a significant determinant of exposure among applicators (Aponso, 2002; Marquart et al., 2003; Bakke et al., 2009; Hines et al., 2011; Phung et al., 2012a; Atabila et al., 2017). Thus, it may be concluded that a direct comparison of the exposure estimates of chlorpyrifos from the whole-body dermal dosimetry method and the urinary TCP method is valid.

Table 1: Field Factors and p-values for Test of Difference in Variables between the Wholebody Dermal Dosimetry and Urinary TCP Methods $(n=16)$.

\begin{tabular}{|l|c|c|c|}
\hline \multicolumn{1}{|c|}{ Field Factor } & $\begin{array}{c}\text { Whole-body Dermal } \\
\text { Dosimetry Method }\end{array}$ & $\begin{array}{c}\text { Urinary TCP } \\
\text { Method }\end{array}$ & p-value \\
\hline Quantity of Insecticide Formulation & 156 & 162 & $0.43 \mathrm{a}$ \\
Applied (median, mL) & 0.4 & 0.6 & $0.04 \mathrm{a}$ \\
Farm Size (median, ha) & 43 & 38 & $0.5 \mathrm{a}$ \\
Crop Height (median, cm) & 53 & 79 & $0.04 \mathrm{~b}$ \\
Spraying Duration (mean, min) & 6 & 13 & $1 \mathrm{c}$ \\
Used PPE (\%) & 63 & 50 & $0.7 \mathrm{c}$ \\
Wore Short Sleeve Shirt (\%) & 100 & 94 & $1 \mathrm{c}$ \\
Wore long trousers (\%) & 50 & 50 & $1 \mathrm{c}$ \\
Occurrence of Leaky Spray Tank (\%) & 81 & 81 & $1 \mathrm{c}$ \\
Occurrence of Spillage (\%) & & & \\
\hline
\end{tabular}

a, Wilcoxon signed ranks test;

b, paired samples t-test;

c, McNemar test 


\subsection{Absorbed Daily Dose of Chlorpyrifos Estimated from the Whole-body Dermal Dosimetry Method (ADD DEM) and Urinary TCP Method (ADDTCP)}

Table 2 shows the descriptive statistics of the Absorbed Daily Dose (ADD) estimates of chlorpyrifos from the whole-body dermal dosimetry method (ADD method $\left(\mathrm{ADD}_{\mathrm{TCP}}\right)$ for the applicators. $\mathrm{ADD}_{\mathrm{DEM}}$ ranged from 5 to $29 \mu \mathrm{g} / \mathrm{kg} / \mathrm{day}$, with a median and a mean of $16 \mu \mathrm{g} / \mathrm{kg} /$ day. For $\mathrm{ADD}_{\mathrm{TCP}}$, the dose estimate ranged from 1 to $71 \mu \mathrm{g} / \mathrm{kg} /$ day, with a median of $5 \mu \mathrm{g} / \mathrm{kg} /$ day and mean of $15 \mu \mathrm{g} / \mathrm{kg} / \mathrm{day}$. Although the median ADD DEM (16 $\mu \mathrm{g} / \mathrm{kg} /$ day) was higher than that for $\operatorname{ADD}_{\text {TCP }}(5 \mu \mathrm{g} / \mathrm{kg} /$ day $)$, the difference was not statistically significant $(p>0.05)$. In addition, the means of ADD DEM $(16 \mu \mathrm{g} / \mathrm{kg} / \mathrm{day})$ and ADD $_{\text {TCP }}(15$ $\mu \mathrm{g} / \mathrm{kg} / \mathrm{day}$ ) were similar. These suggest that, overall, the two methods yielded comparable results in terms of the central tendency measures. The similarity of the two estimates further show that pesticide exposure through the dermal route can be regarded as the most significant in agricultural settings, considering that urinary TCP method incorporates exposure from other routes, such as via inhalation and oral routes.

Table 2: Absorbed Daily Dose of Chlorpyrifos Estimated from Whole-body Dermal Dosimetry (ADD $\left.{ }_{\mathrm{DEM}}\right)$ and Urinary TCP (ADD $\left.{ }_{\mathrm{TCP}}\right)$ Methods for Applicators.

\begin{tabular}{|l|cccc|c|}
\hline & Minimum & Median & Mean & Maximum & p-value \\
\hline $\operatorname{ADD}_{\mathrm{DEM}}(\mu \mathrm{g} / \mathrm{kg} /$ day $)$ & 5 & 16 & 16 & 29 & \\
$\operatorname{ADD}_{\mathrm{TCP}}(\mu \mathrm{g} / \mathrm{kg} /$ day $)$ & 1 & 5 & 15 & 71 & $0.3 \mathrm{a}$ \\
\hline
\end{tabular}

a, Wilcoxon signed ranks test of difference of the median values 
The CPD plots of $\mathrm{ADD}_{\mathrm{DEM}}$ and $\mathrm{ADD}_{\mathrm{TCP}}$ for the individual applicators are shown in Figure 1.

250

251

252

253

254

255

256

257

258

259

260

261

262

263

264

265

266

267

268

269

270

271

The linear regression line of the plot for $\mathrm{ADD}_{\mathrm{DEM}}$ and $\mathrm{ADD}_{\mathrm{TCP}}$ are respectively represented by the following equations:

$$
\mathrm{CP} \%=55 \log \left(\mathrm{ADD}_{\mathrm{DEM}}\right)-98 \quad\left(\mathrm{R}^{2}=0.91\right) \quad \text { Equation } 4
$$

$$
\mathrm{CP} \%=20 \log \left(\mathrm{ADD}_{\mathrm{TCP}}\right)+14 \quad\left(\mathrm{R}^{2}=0.96\right) \quad \text { Equation } 5
$$

The slope of the linear regression line of the plot for $\mathrm{ADD}_{\text {TС }}$ was less steep (20) compared to that of the plot for $\operatorname{ADD}_{\mathrm{DEM}}(55)$. These trends reflect the wide range (1 to $\left.71 \mu \mathrm{g} / \mathrm{kg} / \mathrm{day}\right)$ of

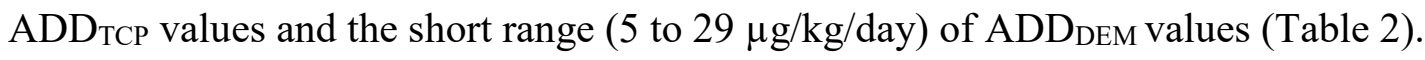

Figure 1 shows that $\mathrm{ADD}_{\mathrm{DEM}}$ values were generally higher than those for $\mathrm{ADD}_{\mathrm{TCP}}$ below the $75^{\text {th }}$ percentile. Conversely, ADD ${ }_{\text {DEM }}$ values were less than those for $A_{D D}{ }_{T C P}$ above the $75^{\text {th }}$ percentile. If the exposure dose estimate from the urinary TCP method is accepted as the more valid then, these findings suggest that the whole-body dermal dosimetry method may overestimate exposure dose at lower percentiles and under-estimate the dose at higher percentiles.

To further investigate the differences trend observed in Figure 1, within the individual applicators, Absorbed Unit Exposure (AUE, \%) values of both the whole-body dermal dosimetry and urinary TCP methods for each applicator were calculated and compared. Based on the assumption that pesticide exposure is proportional to the quantity of active ingredient applied (Sielken Jr, 2005; Beauvais et al., 2007; Hines et al., 2011; Phung et al., 2012b; Atabila et al., 2017), AUE values were calculated as a ratio of the mass of estimated absorbed chlorpyrifos to the mass of chlorpyrifos applied, multiplied by 100 to give AUE as a percentage 
272 (AUE \%). Thus, high absorbed chlorpyrifos corresponds to high AUE value. Plots of the AUE

273

274

275

276

277

278

279 values are shown in Figure 2. With valid estimate of absorbed chlorpyrifos with the wholebody dermal dosimetry method, the resulting AUE values (AUEDEM) and those from the urinary TCP method (AUETCP) for each applicator can be expected to be similar. However, Figure 2 shows that AUEDEM values were higher than AUETCP for applicators A to L ("low exposure" group), reflecting Figure 1 with exposure values below the $75^{\text {th }}$ percentile. On the contrary, the reverse of this trend was true for applicators $\mathrm{N}$ to $\mathrm{P}$ ("high exposure” group), reflecting Figure 1 with exposure values above the $75^{\text {th }}$ percentile.

The above findings also suggest that the whole-body dermal dosimetry was less able to account for variabilities in absorbed doses between applicators. This is mainly because the method assumes that a fixed fraction (4.3\%) of the quantity of insecticide reaching the skin of applicators are absorbed, as demonstrated in the study by Meuling et al. (2005). On the other hand, the urinary TCP method could account for variations in exposure doses between the applicators, which can largely be attributed to differences in the dermal absorption fractions between applicators. Dermal absorption fractions are in turn influenced by differences in the quantity of pesticide exposure on the skin, anatomical site of exposure, skin integrity, skin hydration, age and metabolic rates of applicators (Holmgaard and Nielsen, 2009.).

\subsection{Conclusions}


Overall, the whole-body dermal dosimetry and urinary TCP methods for estimating

chlorpyrifos exposure produced similar estimates, based on the means. However, exposure estimates from the urinary TCP method showed more variation than those from the wholebody dermal dosimetry method. Using the exposure estimates from the urinary TCP method as a comparison, the whole-body dermal dosimetry method appeared to over-estimate exposure doses below the $75^{\text {th }}$ percentile and under-estimate the doses above the $75^{\text {th }}$ percentile.

Nevertheless, the whole-body dermal dosimetry method is valid for providing information regarding the typical levels of pesticide exposure among applicators. The urinary TCP method, which can be difficult at applicator and technical levels, may be used to identify the specific variations in exposure of individuals in a population. Moreover, the whole-body dermal dosimetry method may be used to identify the patterns of pesticide exposure on the anatomical regions of applicators.

\section{Conflict of Interest}

None

\section{Acknowledgements}

This study was supported with funding from Griffith University (Griffith University International Postgraduate Research Scholarship, Griffith University Postgraduate Research Scholarship), Griffith School of Environment (PhD fieldwork fund), Griffith School of Engineering, the Organic Chemistry Department of Queensland Health Forensic and Scientific Services and Dr. Ross Sadler. Special thanks go to the rice farmers of Asutsuare and Akuse 
317 (Ghana), the staff of Kpong Irrigation Scheme (Asutsuare), particularly Albert F. Swatson, 318 Raphael Edifor, Samuel Kwakye and Moses Kodjotse, for their tremendous support during the 319 field work. The authors are also grateful to the staff of the Pesticide Residues Laboratory of 320 Ghana Standards Authority (Accra), Shai-Osudoku District Directorate of the Ministry of Food 321 and Agriculture, Greater Accra Regional Directorate of Ghana Health Service and Osukoku 322 Health Centre (Asutsuare), for their assistance during the fieldwork. 
Albertini, R., Bird, M., Doerrer, N., Needham, L., Robison, S., Sheldon, L. \& Zenick, H. 2006. The Use of Biomonitoring Data in Exposure and Human Health Risk Assessments. Environ. Health Perspect., 114, 1755-1762.

An, X., Ji, X., Wu, M., Hu, X., Yu, R., Zhao, X. \& Cai, L. 2014. Risk Assessment of Applicators to Chlorpyrifos through Dermal Contact and Inhalation at Different Maize Plant Heights in China. J. Agric. Food Chem., 62, 7072-7.

Aponso, G. L. M. 2002. Exposure and Health Risk Assessment for Farmers Occupationally Exposed to Chlorpyrifos in Sri Lanka and Drinking Water and House Dust Analysis for Chlorpyrifos. Doctor of Philosophy Thesis, Oregon State University.

Atabila, A., Phung, D. T., Hogarh, J. N., Osei-Fosu, P., Sadler, R., Connell, D. \& Chu, C. 2017. Dermal Exposure of Applicators to Chlorpyrifos on Rice Farms in Ghana. Chemosphere, 178, 350-358.

Atabila, A., Phung, D. T., Hogarh, J. N., Sadler, R., Connell, D. \& Chu, C. 2018a. Health Risk Assessment of Dermal Exposure to Chlorpyrifos among Applicators on Rice Farms in Ghana. Chemosphere, 203, 83-89.

Atabila, A., Sadler, R., Phung, D. T., Hogarh, J. N., Carswell, S., Turner, S., Patel, R., Connell, D. \& Chu, C. 2018b. Biomonitoring of Chlorpyrifos Exposure and Health Risk Assessment among Applicators on Rice Farms in Ghana. Environ. Sci. Pollut. Res. Int., $10.1007 / \mathrm{s} 11356-018-2259-9$.

Baker, B. A., Alexander, B. H., Mandel, J. S., Acquavella, J. F., Honeycutt, R. \& Chapman, P. 2005. Farm Family Exposure Study: Methods and Recruitment Practices for a Biomonitoring Study of Pesticide Exposure. J. Expo. Anal. Environ. Epidemiol., 15, 491-9.

Bakke, B., De Roos, A. J., Barr, D. B., Stewart, P. A., Blair, A., Freeman, L. B., Lynch, C. F., Allen, R. H., Alavanja, M. C. \& Vermeulen, R. 2009. Exposure to Atrazine and Selected Non-Persistent Pesticides among Corn Farmers During a Growing Season. $J$. Expo. Sci. Environ. Epidemiol., 19, 544-54.

Barr, D. B. \& Angerer, J. 2006. Potential Uses of Biomonitoring Data: A Case Study Using the Organophosphorus Pesticides Chlorpyrifos and Malathion. Environ. Health Perspect., 114, 1763-9.

Barr, D. B., Wilder, L. C., Caudill, S. P., Gonzalez, A. J., Needham, L. L. \& Pirkle, J. L. 2005. Urinary Creatinine Concentrations in the U.S. Population: Implications for Urinary Biologic Monitoring Measurements. Environ. Health Perspect., 113, 192-200. 
Beauvais, S., Powell, S. \& Zhao, W. 2007. Surrogate Handler Exposure Estimates for Use in Assessments by the California Department of Pesticide Regulation. Sacramento, California: California Environmental Protection Agency.

Cocker, J., Mason, H. J., Warren, N. D. \& Cotton, R. J. 2011. Creatinine Adjustment of Biological Monitoring Results. Occup. Med. (Lond.), 61, 349-53.

Damalas, C. A. \& Eleftherohorinos, I. G. 2011. Pesticide Exposure, Safety Issues, and Risk Assessment Indicators. Int. J. Environ. Res. Public Health, 8, 1402-19.

DFS - Department of Food Safety 2006. Analytical Methods for Residual Compositional Substances of Agricultural Chemicals, Feed Additives, and Veterinary Drugs in Food. Japan: Department of Food Safety, Ministry of Health, Labour and Welfare.

Dowling, K. C. \& Seiber, J. N. 2002. Importance of Respiratory Exposure to Pesticides among Agricultural Populations. Int. J. Toxicol., 21, 371-81.

Ellison, C. A., Knaak, J. B., Mcdougall, R. \& Olson, J. R. 2012. Use of Cytochrome P450Specific Parameters and Human Biomarker Data to Develop a Human Physiologically Based Pharmacokinetic/Pharmacodynamic Model for Dermal Chlorpyrifos Exposure. Parameters for Pesticide Qsar and Pbpk/Pd Models for Human Risk Assessment. American Chemical Society.

Farahat, F. M., Ellison, C. A., Bonner, M. R., Mcgarrigle, B. P., Crane, A. L., Fenske, R. A., Lasarev, M. R., Rohlman, D. S., Anger, W. K., Lein, P. J. \& Olson, J. R. 2011. Biomarkers of Chlorpyrifos Exposure and Effect in Egyptian Cotton Field Workers. Environ. Health Perspect., 119, 801-6.

Fenske, R. A., Farahat, F. M., Galvin, K., Fenske, E. K. \& Olson, J. R. 2012. Contributions of Inhalation and Dermal Exposure to Chlorpyrifos Dose in Egyptian Cotton Field Workers. Int. J. Occup. Environ. Health, 18, 198-209.

Fenske, R. A. \& Day, E. W. 2005. Assessment of Exposure for Pesticide Handlers in Agricultural, Residential and Institutional Environments. Occupational and Residential Exposure Assessment for Pesticides. John Wiley \& Sons, Ltd.

Fustinoni, S., Mercadante, R., Polledri, E., Rubino, F. M., Mandic-Rajcevic, S., Vianello, G., Colosio, C. \& Moretto, A. 2014. Biological Monitoring of Exposure to Tebuconazole in Winegrowers. J. Expo. Sci. Environ. Epidemiol., 24, 643-9.

Garabrant, D. H., Aylward, L. L., Berent, S., Chen, Q., Timchalk, C., Burns, C. J., Hays, S. M. \& Albers, J. W. 2009. Cholinesterase Inhibition in Chlorpyrifos Workers: Characterization of Biomarkers of Exposure and Response in Relation to Urinary Tcpy. J. Expo. Sci. Environ. Epidemiol., 19, 634-42. 
Geer, L. A., Cardello, N., Dellarco, M. J., Leighton, T. J., Zendzian, R. P., Roberts, J. D. \& Buckley, T. J. 2004. Comparative Analysis of Passive Dosimetry and Biomonitoring for Assessing Chlorpyrifos Exposure in Pesticide Workers. Ann. Occup. Hyg., 48, 68395.

Health Canada 2014. Re-Evaluation Decision for Propoxur. Ottawa, Ontario: Health Canada Pest Management Regulatory Agency.

Hines, C. J., Deddens, J. A., Coble, J., Kamel, F. \& Alavanja, M. C. 2011. Determinants of Captan Air and Dermal Exposures among Orchard Pesticide Applicators in the Agricultural Health Study. Ann. Occup. Hyg., 55, 620-33.

Holmgaard, R. \& Nielsen, J. B. 2009. Dermal Absorption of Pesticides - Evaluation of Variability and Prevention Environmental Protection Agency, Danish Ministry of the Environment.

Khan, K., Ismail, A. A., Abdel Rasoul, G., Bonner, M. R., Lasarev, M. R., Hendy, O., AlBatanony, M., Crane, A. L., Singleton, S. T., Olson, J. R. \& Rohlman, D. S. 2014. Longitudinal Assessment of Chlorpyrifos Exposure and Self-Reported Neurological Symptoms in Adolescent Pesticide Applicators. BMJ open, 4, e004177.

Marquart, J., Brouwer, D. H., Gijsbers, J. H., Links, I. H., Warren, N. \& Van Hemmen, J. J. 2003. Determinants of Dermal Exposure Relevant for Exposure Modelling in Regulatory Risk Assessment. Ann. Occup. Hyg., 47, 599-607.

Meuling, W. J., Ravensberg, L. C., Roza, L. \& Van Hemmen, J. J. 2005. Dermal Absorption of Chlorpyrifos in Human Volunteers. Int. Arch. Occup. Environ. Health, 78, 44-50.

Mohabbati-Kalejahi, E., Azimirad, V., Bahrami, M. \& Ganbari, A. 2012. A Review on Creatinine Measurement Techniques. Talanta, 97, 1-8.

Nolan, R. J., Rick, D. L., Freshour, N. L. \& Saunders, J. H. 1984. Chlorpyrifos: Pharmacokinetics in Human Volunteers. Toxicol. Appl. Pharmacol., 73, 8-15.

OECD - Organisation for Economic Co-Operation and Development 1997. Guidance Document for the Conduct of Studies of Occupational Exposure to Pesticides During Agricultural Application. Paris: OECD Environmental Health and Safety Publications.

Phung, D. T., Connell, D., Miller, G. \& Chu, C. 2012a. Probabilistic Assessment of Chlorpyrifos Exposure to Rice Farmers in Viet Nam. J. Expo. Sci. Environ. Epidemiol., $22,417-23$.

Phung, D. T., Connell, D., Miller, G., Hodge, M., Patel, R., Cheng, R., Abeyewardene, M. \& Chu, C. 2012b. Biological Monitoring of Chlorpyrifos Exposure to Rice Farmers in Vietnam. Chemosphere, 87, 294-300. 
Putnam, R. A. \& Clark, J. M. 2007. Dosimetry and Biomonitoring Following Golfer Exposure to Chlorpyrifos. Assessing Exposures and Reducing Risks to People from the Use of Pesticides. American Chemical Society.

Ross, J., Chester, G., Driver, J., Lunchick, C., Holden, L., Rosenheck, L. \& Barnekow, D. 2008. Comparative Evaluation of Absorbed Dose Estimates Derived from Passive Dosimetry Measurements to Those Derived from Biological Monitoring: Validation of Exposure Monitoring Methodologies. J. Expo. Sci. Environ. Epidemiol., 18, 211-30.

Saieva, C., Aprea, C., Tumino, R., Masala, G., Salvini, S., Frasca, G., Giurdanella, M. C., Zanna, I., Decarli, A., Sciarra, G. \& Palli, D. 2004. Twenty-Four-Hour Urinary Excretion of Ten Pesticide Metabolites in Healthy Adults in Two Different Areas of Italy (Florence and Ragusa). Sci. Total Environ., 332, 71-80.

Scher, D. P., Alexander, B. H., Adgate, J. L., Eberly, L. E., Mandel, J. S., Acquavella, J. F., Bartels, M. J. \& Brzak, K. A. 2007. Agreement of Pesticide Biomarkers between Morning Void and 24-H Urine Samples from Farmers and Their Children. J. Expo. Sci. Environ. Epidemiol., 17, 350-7.

Sexton, K., Needham, L. \& Pirkle, J. 2004. Human Biomonitoring of Environmental Chemicals. Am. Sci., 92, 38 - 45.

Sielken Jr, R. L. 2005. Probabilistic Approaches to Aggregate and Cumulative Risk Assessment. In: Roberts, T., Miyamoto, J., Franklin, C. A. \& Worgan, J. P. (eds.) Occupational and Residential Exposure Assessment for Pesticides.

WHO - World Health Organization 1982. Field Surveys of Exposure to Pesticides Standard Protocol. Geneva: World Health Organization. 